\title{
INSPECCIÓN AL CICLO DE VIDA DE PROYECTOS DE CONSTRUCCIÓN SOSTENIBLE: HACIA UNA HOJA DE RUTA BASADA EN BIOMIMETISMO Y ECONOMÍA CIRCULAR
}

\author{
Beermann, Kimberly1,2 \\ 1 Universidad Tecnológica de Panamá \\ 2 International Association for Hydro-Environment Engineering and Research (IAHR) Panama Young \\ Professionals Network \\ Ciudad de Panamá, Panamá \\ email: kimberly.beermann@utp.ac.pa
}

\begin{abstract}
The construction sector is one of the most influential sectors impacting the economy and is expected to expand due to population growth of almost $300 \%$ by 2050 , according to the National Energy Plan. However, it must work on the transition towards sustainability and resilience in the face of climate change since its growth implies a high consumption of resources and contribution of greenhouse gases. The need to establish practices and strategies that encompass the dimensions of sustainability (environment, society, and economy) and work towards a circular economy is imminent. Currently, there is little guidance in the framework beyond certifications as well as in planning, management, and evaluation tools for implementation. Different studies vary in the number of phases and considerations for projects. Therefore, the research work proposes the development of a unified roadmap, with defined phases, practices, and indicators based on principles inspired by nature such as biomimicry (Greek words: "bio" means life and "mimesis", imitation) and focused on a circular economy, which was validated by construction professionals where strengths, opportunities, skills, and threats were identified with a high level of acceptance. This contributes to strengthen the field of sustainable construction project management and sets a precedent for Panama with the innovative approaches of biomimicry and circular economy.
\end{abstract}

Keywords: biomimicry, circular economy, project management, roadmap, sustainable construction. 


\section{Resumen}

El sector de la construcción es uno de los más influyentes que impactan en la economía, y se prevé su expansión a causa del crecimiento de la población de casi $300 \%$ al 2050 , según el Plan Energético Nacional. Sin embargo, este debe trabajar en la transición hacia la sostenibilidad y resiliencia frente al cambio climático, ya que su crecimiento implica un alto consumo de recursos y contribución de gases de efecto invernadero. La necesidad de establecer prácticas y estrategias que abarquen la dimensionalidad de la sostenibilidad (ambiente, sociedad y economía) y que se trabaje por una economía circular, son inminentes. Actualmente se cuenta con poca orientación en el marco de referencia más allá de certificaciones, así como en planificación, gestión y herramientas de evaluación para su implementación. En diferentes estudios se varía el número de fases y consideraciones para los proyectos. Por eso el trabajo de investigación propone el desarrollo de hoja de ruta unificada, con definición fases, prácticas e indicadores basados en principios inspirados en la naturaleza como la biomímesis (palabras griegas: "bio" significa vida y "mimesis", imitación) y enfocados en una economía circular, la cual fue validada por profesionales de la construcción donde se identificaron las fortalezas, oportunidades, destrezas y amenazas contando con un alto nivel de aceptación. Esto contribuye a fortalecer el campo de la gestión de proyectos de construcción sostenible y dejar un precedente para Panamá con los enfoques innovadores de la biomímesis y economía circular.

Palabras claves: biomímesis, construcción sostenible, economía circular, gestión de proyectos, hoja de ruta.

\section{INTRODUCCIÓN}

La industria de la construcción presenta una gran importancia para la economía al igual que oportunidades ante retos de cambio climático y desafíos globales [1]. Por esto se tiene la necesidad de que se tomen acciones que abarquen aspectos ambientales, sociales y económicos cumpliendo con la dimensión de la sostenibilidad (triple resultado) en el proceso del ciclo de vida del proyecto y gestión [2]. Además que en resultados se ha comprobado la rentabilidad e importancia de inversión en fases iniciales así como los ahorros en operatividad, donde se tienen los edificios con una reducción al $50 \%$ en energía y mayor durabilidad [3]. Se prevé su expansión a un $50 \%$ en escala global, debido al crecimiento poblacional y demanda de edificaciones y energía [4], donde se incluye a Panamá con un pronóstico de aumento demográfico alrededor del 300\% al 2050 según el 
Plan Energético Nacional [5].

Actualmente existe una literatura diversa donde se reconoce la relevancia del tema, pero con enfoques en distintas direcciones como cualidades de la planificación, propuestas preliminares para toma de decisiones, esquematización de procesos y la presencia de los sistemas de calificación de edificios verdes (LEED, BREAM, entre otros) así como normas internacionales, que han sido objeto de debate por el alcance en totalidad de la sostenibilidad. Por lo cual, esta investigación pretende proponer y evaluar una hoja de ruta con definición de fases, prácticas e indicadores bajo el triple resultado (de Triple Bottom Line: profit, people and planet), economía circular y biomímesis, con miras a unificar la definición y criterios de proyectos de construcción sostenible. Siendo estos últimos factores fundamentales para emular a la naturaleza en una transición de economía linear, a circular y a resolver los desafíos de la construcción, para lograr ser sostenible.

\section{METODOLOGÍA}

La Biomímesis es el estudio y la inspiración en componentes biológicos y procesos naturales, para resolver problemáticas entendiendo funciona de forma autosostenible [6], [7]. Como referencia, se utiliza el "Enfoque basado en el problema", donde a través de un comportamiento, función o característica de un organismo o ecosistema, se soluciona el problema y la metodología de diseño biomimética "BioGen" la cual se divide en dos fases: diseño preliminar y emulación [8]. Como problema, se presenta la diversidad de enfoques en la construcción sostenible y sus procesos. Teniendo como desafíos el triple resultado: ambiente, economía y sociedad, y la integración de economía circular como característica para el análisis de pináculos.

\section{A. Fase de Diseño Preliminar}

Consiste en realizar una revisión de organismos y ecosistemas a través de la página AskNature en base a funciones, procesos, y factores en entidades biológicas llamadas pináculos en un modelo exploratorio. Estos cuentan con soluciones a los desafíos identificados para la metodología, como el de utilizar materiales locales, reutilizar materiales para la construcción, compensar ecológicamente o realizar ahorros de energía y agua. Luego se realiza un análisis en base a las estrategias y características principales, para la matriz de análisis de pináculos y la matriz de ruta de diseño. Donde la última permite la inclusión de la economía circular entre sus categorías. En las acciones de esta categoría, se incluyen la del marco ReSOLVE [9]: regenerar, compartir, optimizar, intercambiar y ciclo. A través de la última matriz se obtienen las características dominantes para cada categoría contemplando los desafíos de la sostenibilidad: protección del ambiente, bienestar social y armonización económica; así como el de alcanzar una economía circular en la construcción. En esta se busca atribuir a un pináculo imaginario la característica más dominante por categoría y 
definir su trayectoria correspondiente, con la finalidad de reducir la complejidad de las soluciones encontradas [8].

\section{B. Fase de Emulación}

En esta se transforman los resultados de la matriz de ruta de diseño, en conceptos el cual sería el modelo biocircular: activo, comportamiento, vivienda y compartir. Su validación se da a través de una encuesta a 30 profesionales de la construcción, con diferentes cargos y años de experiencia, para conocer sus conocimientos en los conceptos desarrollados, niveles de aceptación de las definiciones e inclusión del modelo biorcircular así como la identificación de oportunidades y barreras, bajo el contexto de Panamá.

\section{RESULTADOS}

Como resultado se tiene la influencia del Modelo Biocircular a partir de la matriz ruta de diseño, donde de destacaron los conceptos: Activo (A), que define que los procesos sean dinámicos, eficientes y eficaces; Comportamiento (B), considerando: conocimiento técnico, dimensión humana y administración; Vivienda $(\mathrm{H})$, en base al cumplimiento de objetivos del proyecto y de sostenibilidad. Por último, el enfoque de economía circular, donde se tuvo la acción de Compartir (S), se involucra la reutilización, reciclaje, durabilidad a través de diseño y mantenimiento, así como la optimización de procesos. Esta complementa la definición de fases, las cuales son: inicio, planificación, diseño, construcción, operación y mantenimiento, y entrega (figura 1). También la evaluación de indicadores encontrados en la literatura de construcción sostenible y tecnologías de apoyo, como el "Modelo de Información de la Construcción" (BIM por sus siglas en inglés) y Sistemas de Información Geográfica.

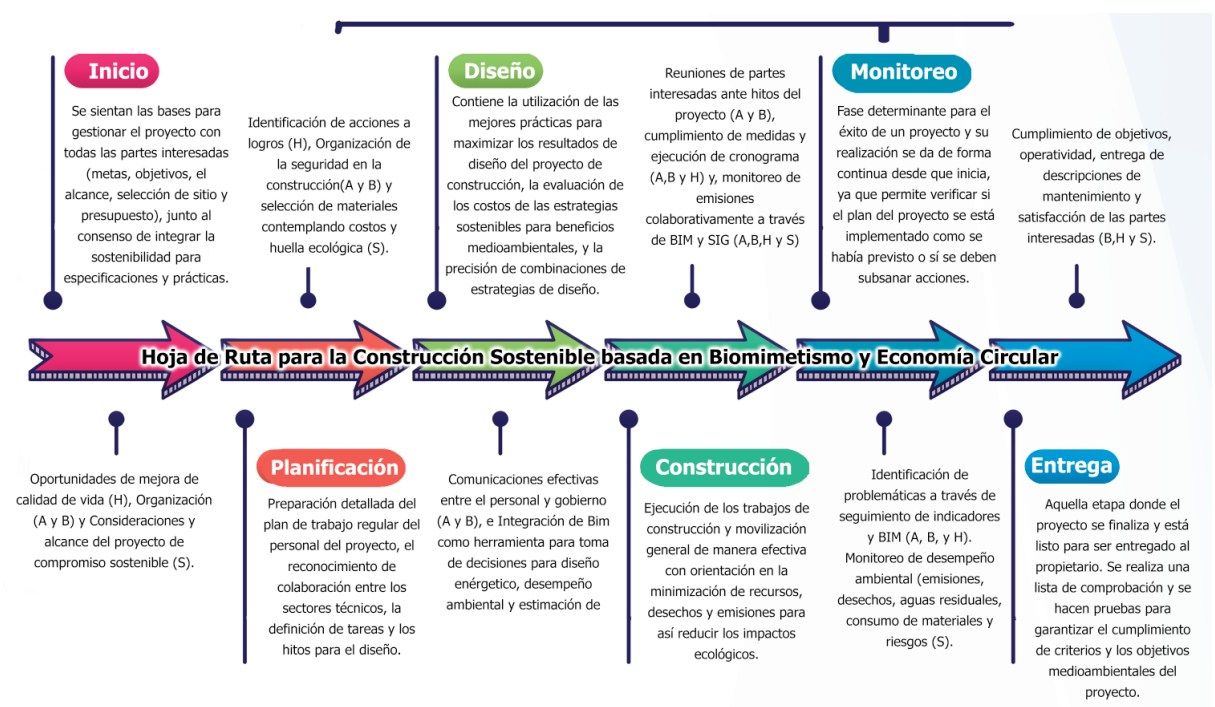

Figura 1. Resumen de definición de fases de la Hoja de Ruta con los conceptos del modelo biocircular. 
Para la validación a través de la encuesta, se tuvo mayor concurrencia de profesionales con 5 a 10 años de experiencia y con un conocimiento medio en temas de construcción sostenible, economía circular y biomímesis. En términos generales, las definiciones de la hoja de ruta con el enfoque biocircular han obtenido un $83 \%$ de aceptación. Las recomendaciones más destacadas son: la sensibilización del tema a diseñadores, constructores e inversionistas; considerar normas de diseño y seguridad de materiales y, contemplar los retos de colaboración entre diferentes contratistas y la información gestionada por cada uno.

Incluyendo un análisis de fortalezas, oportunidades, destrezas y amenazas en el contexto de Panamá, destacan como desafíos: el desconocimiento de construcción sostenible, la falta de apoyo gubernamental y las actitudes humanes ante el cambio. En la percepción de fortalezas, sobresalen los beneficios de reputación, automatización de estrategias y la optimización de residuos, así como las oportunidades de ahorros e ingresos económicos al igual que el desarrollo de competencias del recurso humano en sostenibilidad.

\section{CONCLUSIONES}

Con el objetivo de proponer y evaluar una hoja de ruta con definición de fases, prácticas e indicadores, con miras a unificar la definición y criterios de proyectos de construcción sostenible, este estudio se posiciona como un gran complemento en el campo de la gestión de proyectos y construcción sostenible. Esto último, por su innovación con la inclusión del enfoque biomimético y la enfatizada búsqueda de lograr una economía circular en definiciones y consideraciones del proceso de la construcción. Teniendo beneficios trascendentales para el ambiente hasta inversionistas, diseñadores y contratista, por la optimización y beneficios de tener la sostenibilidad como un factor de éxito, junto a los costos, tiempo y respuesta a riesgos.

\section{Referencias}

[1] S. E. Helmy and M. M. Aboulnaga, "Future Cities: The Role of Biomimicry Architecture in Improving Livability in Megacities and Mitigating Climate Change Risks," in Sustainable Ecological Engineering Design, Springer International Publishing, 2020, pp. 35-49.

[2] G. Silvius, "Making Sense of Sustainable Project Management," Ann. Soc. Sci. Manag. Stud., vol. 2, no. 4, Jan. 2019, doi: 10.19080/asm.2019.02.555594.

[3] K. H. Al Rumaithi and S. M. Beheiry, "A framework for green project management processes in construction projects," Int. J. Sustain. Soc., vol. 8, no. 2, pp. 126-144, 2016, doi: 10.1504/ IJSSOC.2016.077514.

[4] L. Liang et al., "Rectify the performance of Green Building Rating Tool (GBRT) in sustainability: Evidence from ISO 21929-1," J. Clean. Prod., vol. 278, p. 123378, 2021, doi: 10.1016/j. jclepro.2020.123378.

[5] Secretaria Nacional de Energía, “Un sistema energético en transición - Plan Energético 
Nacional," 2017. [Online]. Available: http://www.energia.gob.pa/energia/wp-content/uploads/ sites/2/2018/04/PEN-2017-Versión-Final.pdf.

[6] M. Chen Austin, D. Garzola, N. Delgado, J. U. Jiménez, and D. Mora, "Inspection of biomimicry approaches as an alternative to address climate-related energy building challenges: A framework for application in Panama," Biomimetics, vol. 5, no. 3, 2020, doi: 10.3390/BIOMIMETICS5030040.

[7] M. Bankar and V. Jogdand, "Feasibility Study of Adaptation of Biomimicry Approach in Green Building," E3S Web Conf., vol. 170, 2020, doi: 10.1051/e3sconf/202017006010.

[8] Q. Lv, O. Dqg, S. Fruehwirth, and L. I. Development, LIVING envelope, vol. 113. 2008.

[9] R. Gower and P. Schröder, Virtuous circle: How the circular economy can create jobs and save lives in low and middle-income countries, no. August. 2016.

\section{Autorización y Licencia CC}

Los autores autorizan a APANAC XVIII a publicar el artículo en las actas de la conferencia en Acceso Abierto (Open Access) en diversos formatos digitales (PDF, HTML, EPUB) e integrarlos en diversas plataformas online como repositorios y bases de datos bajo la licencia CC: Attribution-NonCommercial-ShareAlike 4.0 International (CC BY-NC-SA 4.0) https://creativecommons.org/ licenses/by-nc-sa/4.0/.

Ni APANAC XVIII ni los editores son responsables ni del contenido ni de las implicaciones de lo expresado en el artículo. 